\title{
Diffusion pathways of hydrogen across the steps of a vicinal $\mathrm{Si}(001)$ surface
}

\author{
M. Lawrenz, ${ }^{1}$ P. Kratzer, ${ }^{2,3}$ C. H. Schwalb,${ }^{1}$ M. Dürr, ${ }^{1,4}$ and U. Höfer ${ }^{1}$ \\ ${ }^{1}$ Fachbereich Physik und Zentrum für Materialwissenschaften, Philipps-Universität, D-35032 Marburg, Germany \\ ${ }^{2}$ Fritz-Haber-Institut der Max-Planck-Gesellschaft, Faradayweg 4-6, D-14195 Berlin, Germany \\ ${ }^{3}$ Fachbereich Physik, Universität Duisburg-Essen, D-47048 Duisburg, Germany \\ ${ }^{4}$ Fakultät Angewandte Naturwissenschaften, Hochschule Esslingen, D-73728 Esslingen, Germany
}

(Received 5 January 2007; published 26 March 2007)

\begin{abstract}
Hydrogen diffusion across $D_{B}$ steps on $\mathrm{Si}(001)$ surfaces is investigated by means of variable-temperature scanning tunneling microscopy and first-principles calculations. Experimentally, the hopping rate for diffusion from the step sites to the Si dimers of the upper terrace was found to be more than one order of magnitude higher than that for diffusion to the lower terrace. This clear preference, opposite to the trend for the respective binding energies, is explained by first-principles calculations that identify a metastable intermediate to be responsible for the unexpected lowering of the energy barrier for upward diffusion.
\end{abstract}

DOI: 10.1103/PhysRevB.75.125424

\section{INTRODUCTION}

Surface diffusion is an important elementary step in many technological processes, such as crystal growth or reactive surface etching. With the possibility to follow single atomic events by microscopy, much insight has been gained into the complex microscopic mechanisms governing these processes. In particular, scanning tunneling microscopy (STM) studies have contributed much to the atomistic understanding of diffusion processes on semiconductor surfaces. However, the complexity of real surfaces (due to steps, kinks, etc.) and the possibly important role of rare events leaves ample room for further exploration. This is exemplified by state-of-the-art simulations of crystal growth, where for most materials the simple solid-on-solid model ${ }^{1}$ still prevails due to the lack of more detailed microscopic information that could be input to the simulations. This widely used approach relies on the assumption that the activation energy for a process depends only on its initial state, or, more generally, on a linear combination of the initial and final state energies. Only in a few cases, e.g., self-diffusion on metal surfaces, ${ }^{2}$ this assumption has been tested theoretically. In situations where steps play an important role, e.g., for the step-flow growth mode of silicon, it is common practice to add the relevant processes in a phenomenological way to the growth model. ${ }^{3}$ Alternatively, calculated barrier heights from first-principles calculations for Si diffusion across steps could be used. ${ }^{4}$

In the present paper, we show that the assumed proportionality of energy barriers and binding energies cannot be generalized to step diffusion at semiconductor surfaces. Real-space, real-time measurements of hydrogen diffusion at step sites of $\mathrm{Si}(001)$ show a clear preference of diffusion from the steps to the upper terrace although the binding energy on the upper terrace is lower than on the lower terrace. This observation is in contradiction to common assumptions about the scaling of diffusion barriers. However, it can be explained by calculations using density-functional theory: It is found that the geometric and electronic configurations near the steps support a metastable intermediate state along the diffusion path from the step to the upper terrace, which reduces the diffusion barrier. Such an intermediate state is absent for diffusion to the lower terrace.

Hydrogen diffusion on the flat $\mathrm{Si}(001)$ surface has been in the focus of previous STM studies. ${ }^{5-7}$ Activation energies for
PACS number(s): 68.43.Jk, 68.37.Ef, 71.15.Mb, 82.65.+r

hopping between the two dangling bonds of one dimer [1.0 eV (Ref. 7)] and along the dimer rows [1.7 eV (Ref. 5)] were determined and found to be in qualitative agreement with theoretical results. ${ }^{8-10}$ Diffusion from one dimer row to the next is not observed experimentally due to the highenergy barrier for this process. ${ }^{8-10}$ Steps perpendicular to the dimer rows are expected to hinder the quasi-one-dimensional diffusive motion along the rows, i.e., hopping across steps is a rare event. While STM studies of diffusion were so far limited to relatively frequent events, we succeeded to extend this technique to the rare hopping events across steps by exploiting the adsorption properties of the $\mathrm{H} / \mathrm{Si}(001)$ system. Dissociative adsorption of molecular $\mathrm{H}_{2}$ has a much lower activation barrier at double-height $D_{B}$ step sites than at terrace sites. ${ }^{11-14}$ As a consequence, at room temperature $\mathrm{H}_{2}$ adsorption is highly site selective for double-height steps and it is possible to fully decorate the steps with atomic hydrogen while keeping the terraces free. ${ }^{15,16}$ Hydrogen diffusion away from the step sites can then be imaged in real time with a variable-temperature STM at surface temperatures of $550 \mathrm{~K}$ and higher. In this way, the various single atomic hopping processes that occur at step sites can be identified and the corresponding microscopic diffusion rates can be determined.

\section{EXPERIMENT}

The measurements presented here were performed using a commercial variable-temperature STM (Omicron VT-STM). The experimental setup is enclosed in an ultrahigh vacuum chamber with a base pressure of about $5 \times 10^{-11}$ mbar. All experiments were performed with $n$-doped vicinal $\mathrm{Si}(001)$ samples cut under an angle of $5.5^{\circ} \pm 0.5^{\circ}$ toward the (110) direction. The $\mathrm{Si}(001)$ surfaces were prepared by resistive current heating. ${ }^{16}$ After the preparation, the surface shows a $(2 \times 1)$ structure and consists of terraces separated by straight steps two atomic layers in height, known as $D_{B}$ steps. ${ }^{17}$ The surface temperature was calibrated against the heating current by using a $K$-type thermocouple, which was glued onto the sample after a complete cycle of experiments. Hydrogen gas $(99.9999 \%$ purity) was dosed via a gas inlet system 


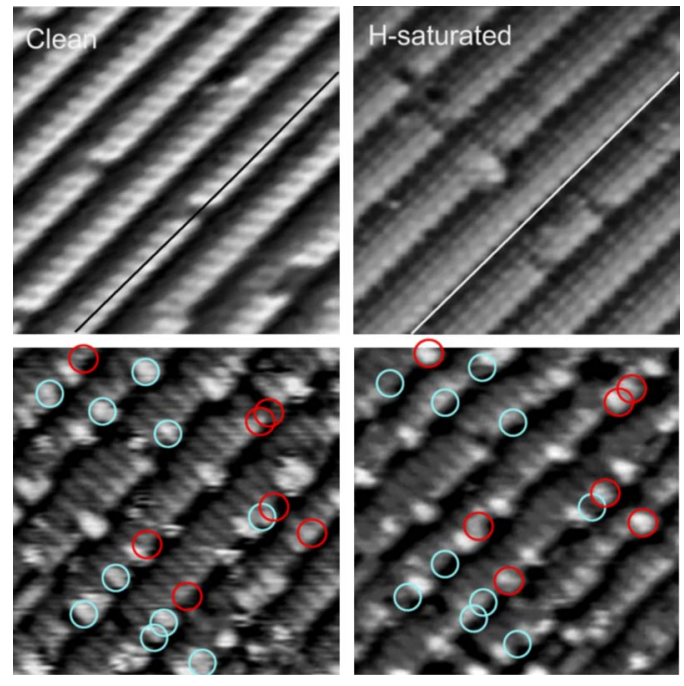

FIG. 1. (Color online) Upper row: STM-images of the $\mathrm{Si}(001)$ surface with clean (left) and hydrogen-saturated $D_{B}$ steps (right). Hydrogen adsorption is seen to suppress the bright features associated with the dangling bonds of the step sites. The black bar in the left and the white bar in the right image indicate the upper edge of a terrace; the step saturation is $98 \%$. Second row: subsequently acquired STM images of the same area $\left(100 \times 100 \AA^{2}\right)$ at $T_{s}$ $=568 \mathrm{~K}$. Hydrogen diffusion away from the H-saturated, dark step sites in the left image to the first dimer on the terrace can be seen as a newly appearing bright spot in the right image and is marked by red (dark gray) circles. Hopping from this configuration back to the step site is identified by the disappearance of these features marked by blue (light gray) circles.

equipped with a liquid nitrogen trap to freeze out residual $\mathrm{H}_{2} \mathrm{O}$.

The quality of the surface preparation was checked by scanning areas of the clean $\mathrm{Si}(001)$ surface as large as 400 $\times 400 \AA^{2}$. A tunneling voltage $U=-2.1 \mathrm{~V}$ and tunneling currents from $I=-0.21 \mathrm{nA}$ to $I=-0.44 \mathrm{nA}$ were employed to acquire filled-state images. Empty-state images were measured under conditions of $U=+0.8 \mathrm{~V}$ and similar tunneling currents; the tunneling conditions were carefully adjusted not to induce movements of the adsorbates by the scanning process itself. An example is shown in Fig. 1, where the $D_{B}$ steps can be easily identified. With a hydrogen exposure of $1350-1500 \mathrm{~L}\left(1 \mathrm{~L}=1.33 \times 10^{-6}\right.$ mbar $\left.\mathrm{s}\right)$, the selective adsorption at the $D_{B}$ sites leads to hydrogen saturated step edges but otherwise clean $\mathrm{Si}(001)$. A maximum step saturation of $99 \%$ was obtained. This saturation of the step sites is observed both in the filled-state images (negative sample bias) and unfilled-state images (positive sample bias) by the disappearance of the bright features at the step sites. ${ }^{16}$ Since different configurations of the hydrogen atoms at the step sites can be distinguished more easily in empty-state images, these images are used exclusively in the following discussion.

For the real-time diffusion measurements, successive scans of the same area of the surface after definite time intervals of $92 \mathrm{~s}$ were performed at $568 \mathrm{~K}$. As shown in Fig. 1 (lower panels), representative regions of the $\mathrm{Si}(001)$ surface could be relocated and the configurations near the step edge were analyzed. It is obvious that most of the step sites remain saturated (black step sites). Information on the diffusion processes was obtained from the sites where the brightness changes between two subsequent images.

\section{THEORY}

In order to understand the microscopic mechanism of hydrogen diffusion across Si step edges, especially the experimentally observed asymmetry between step-up and stepdown diffusion (see below), we performed first-principles total-energy calculations for various atomic configurations. We used density-functional theory (DFT) in conjunction with pseudopotentials and a plane-wave basis set. ${ }^{18}$ The exchange-correlation functional was treated by the generalized-gradient approximation (GGA). ${ }^{19}$ For silicon, we generated an $a b$ initio, norm-conserving pseudopotential, ${ }^{20}$ while the bare Coulomb potential was used for hydrogen. We performed transition state searches for $\mathrm{H}$ diffusion in the configuration space of the $\mathrm{H}$ atoms and the $\mathrm{Si}$ atoms of the four topmost layers using the ridge method. ${ }^{21}$ In addition, we checked that the found transition state indeed connects the intended potential minima by performing unconstrained relaxations of the atomic positions for starting configurations slightly displaced to either side of the transition state. All calculations, including geometry optimizations, were performed with a basis set of plane waves up to a cutoff energy of 30 Ry. For the barrier heights reported below, the calculations were repeated with the same geometries, but with a cutoff of $40 \mathrm{Ry}$. As geometrical model for the $D_{B}$ step, vicinal surfaces with Miller indices ( $\left.\begin{array}{llll}1 & 1 & 7\end{array}\right)$ or ( $\left.\begin{array}{lll}1 & 1 & 1\end{array}\right)$ were employed by using a monoclinic supercell for the calculations. In this geometry, periodically repeated rebonded $D_{B}$ steps are separated by terraces with two or four $\mathrm{Si}$ dimers, respectively. Four $\mathbf{k}$ points in the irreducible part of the Brillouin zone were used for $\mathbf{k}$-space integration.

In order to support the assignment of observed STM images to atomic configurations, the output of the DFT calculations was used to generate calculated STM images, using the Tersoff-Hamann approximation. ${ }^{22}$ For simulating the empty-state images, the unoccupied electronic density of states was integrated in an energy interval between the top of the valence band and $0.7 \mathrm{eV}$ above it. Subsequently, the isocontour surface of this integrated density of states was plotted at a value of $0.3 \times 10^{-7}$ bohr $^{-3}$ above the surface and compared to the experimental STM images. This calculational procedure corresponds to an infinitely sharp, $\delta$-functionlike tip.

\section{EXPERIMENTAL RESULTS}

The first step in the analysis of the changes between successive STM images is the identification of the relevant configurations. In addition to a comparison with previous experimental results for $\mathrm{H}$ adsorption on $\mathrm{Si}(001),{ }^{16,23,24}$ relevant configurations were identified by comparing to simulated empty-state STM images, as shown in Fig. 2. Note that the simulated STM images were calculated for supercells with a period of four lattice constants (two dimer rows) in the di- 

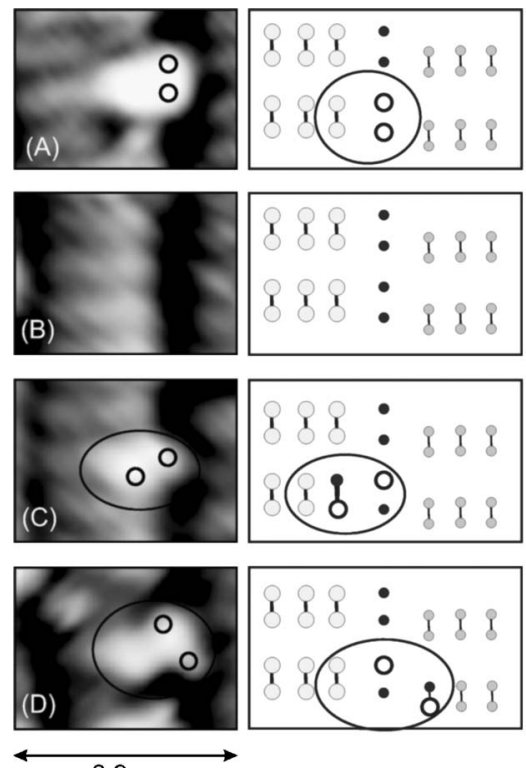

$3.9 \mathrm{~nm}$
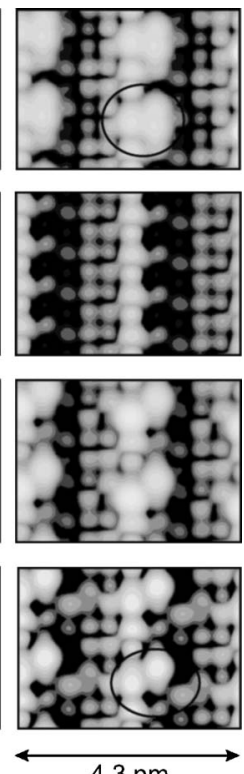

$4.3 \mathrm{~nm}$

FIG. 2. Measured (left panels) and calculated (right panels) STM images for different configurations of hydrogen at the step edge. In the middle, the situation is sketched with occupied Si atoms (dark gray) and bare Si atoms (light gray and white). The upper terrace is in the left part of the images. The saturated step (B) can clearly be distinguished from a pair of unsaturated step sites (A). One hydrogen atom has moved to the adjacent Si dimer of the upper terrace in (C) and to the lower Si dimer in (D).

rection parallel to the step, whereas the experimental images show single, isolated features. In the experimental images, a saturated step site as in (B) can be clearly distinguished from the completely $\mathrm{H}$-free step site (A). The former appears dark, while the latter shows a very bright feature due to two dangling bonds. The calculated images support this interpretation, demonstrating that the STM is mainly sensitive to the electronic density of states in the band gap introduced by the dangling bonds. While saturating the dangling bond of a $\mathrm{Si}$ atom in a Si dimer by a hydrogen atom quenches its density of states, the persisting dangling bond at the other $\mathrm{Si}$ atom of the dimer appears brighter than usual. This dangling bond is shifted up in energy and partly depleted of charge when the $\mathrm{H}$ atom adsorbs on the neighboring $\mathrm{Si}$ atom and therefore becomes more pronounced in an empty-state STM image. This effect allows us to identify motions of the $\mathrm{H}$ atoms, both in the experimental and in the simulated STM images. Since the simulated STM images correspond to an "unrealistically sharp" tip, more detailed features are visible in the simulations, but a comparison of the brightest features only allows for a consistent interpretation. In (C), a configuration is shown where one hydrogen atom is bound to the step edge and another one to the adjacent dimer of the upper terrace. We note that $(\mathrm{C})$ is the configuration which is expected after a hydrogen hopping event to the upper terrace, i.e., step-up diffusion. It is similar to the configuration after a hopping event from the step site onto the lower terrace, i.e., stepdown diffusion, shown in (D). However, for the latter one the hydrogen atom on the lower terrace induces another bright spot on the respective dimer row on the lower terrace. This allows for a clear distinction between step-up and step-down diffusion.

Analyzing images like those in Fig. 1, the preferred diffusion pathway for atomic hydrogen away from the $D_{B}$ step sites can be identified: Hopping from the steps onto the upper terrace is strongly preferred. Additionally, we observe a high probability for the hydrogen atom to diffuse back to the step sites: In the STM images, back diffusion from the upper terrace can be identified by the disappearance of feature (C) between two successive images (see Fig. 1). A first estimate for the hopping rates (e.g., for the step-up diffusion rate) can be obtained by counting the number of all identified events, e.g., where a step site changes from type (B) to (C), and by dividing by the number of initially occupied sites and the elapsed time $t=92 \mathrm{~s}$. With 193 events out of 1912 initial configurations for diffusion from the steps to the upper terraces, we calculated a diffusion rate of $1.1 \times 10^{-3} \mathrm{~s}^{-1}$ for this process. Likewise, this simple analysis leads to diffusion rates of $3.1 \times 10^{-3} \mathrm{~s}^{-1}$ for the diffusion back from the upper terrace onto the steps and $2.45 \times 10^{-3} \mathrm{~s}^{-1}$ for diffusion along the dimer rows. Diffusion of $\mathrm{H}$ atoms from the step sites to the lower terraces is found to have the smallest rate: Only eight of such events could be observed for this diffusion process leading to a rate of $4.5 \times 10^{-5} \mathrm{~s}^{-1}$.

For the given diffusion rates associated with diffusion onto the upper terrace and back to the step edge, there exists a non-negligible probability that the $\mathrm{H}$ atoms perform multiple hops between two $92 \mathrm{~s}$ scans. For a more accurate treatment of the step-up diffusion rate, we therefore consider a system of three coupled rate equations describing the population of the step site $P_{s}$, of the upper terrace edge site $P_{\text {te }}$, and of an adjacent "regular" terrace site $P_{\mathrm{tr}}$. Diffusion from the step to the lower terrace can safely be neglected in this analysis because of its very low diffusion rate,

$$
\begin{aligned}
d P_{s} / d t= & -R_{\text {step } \rightarrow \text { up }} P_{s}\left(1-P_{\mathrm{te}}\right)+R_{\text {terrace } \rightarrow \text { step }} P_{\text {te }}\left(1-P_{s}\right), \\
d P_{\mathrm{te}} / d t= & \left(R_{\text {step } \rightarrow \text { up }} P_{s}+R_{\text {terrace }} P_{\mathrm{tr}}\right)\left(1-P_{\mathrm{te}}\right) \\
& -\left[R_{\text {terrace } \rightarrow \text { step }}\left(1-P_{s}\right)+R_{\text {terrace }}\left(1-P_{\mathrm{tr}}\right)\right] P_{\mathrm{te}}, \\
d P_{\mathrm{tr}} / d t= & R_{\text {terrace }}\left[P_{\mathrm{te}}\left(1-P_{\mathrm{tr}}\right)-P_{\mathrm{tr}} /\left(1+R_{\mathrm{terrace}} t\right)\right] .
\end{aligned}
$$

We solve the coupled rate equations for two different initial conditions, i.e., initial occupation of the step site alone, $P_{s}$ $=1, P_{\mathrm{te}}=0$, and $P_{\mathrm{tr}}=0$, and initial occupation of only the upper terrace edge site, $P_{\mathrm{te}}=1, P_{s}=0$, and $P_{\mathrm{tr}}=0$. For the rate constant for terrace diffusion $R_{\text {terrace, }}$, we used the value as determined by direct counting these events, i.e., 2.45 $\times 10^{-3} \mathrm{~s}^{-1}$ at $T=568 \mathrm{~K}$. This rate might be a slight underestimation due to multiple hopping events as well. However, the difference between this value and the diffusion rates reported for the flat $\operatorname{Si}(001)$ surface $\left[12 \times 10^{-3} \mathrm{~s}^{-1}\right.$ (Ref. 5)] might be rather attributed to the differences in the barrier heights on the six-dimer-wide terraces of the vicinal surface and on flat $\mathrm{Si}(001)$. The hitherto unknown rate constants for hopping from the step to the upper terrace $R_{\text {step } \rightarrow \text { up }}$ and for the reverse process $R_{\text {terrace } \rightarrow \text { step }}$ are then obtained by fitting the appropriate solution of the rate equations over the time interval of the measurement, $t=92 \mathrm{~s}$, to the measured prob- 
TABLE I. Experimentally derived rates at $568 \mathrm{~K}$ and estimated barriers, as well as energy barriers from first-principles calculations for elementary hops. The error bars in the experimental values describe the statistical error as estimated from the square root of the counts. The values for $R_{\text {step } \rightarrow \text { up }}$ and $R_{\text {terrace } \rightarrow \text { step }}$ were derived by analysis via the coupled rate equations.

\begin{tabular}{lccc}
\hline \hline$T=568 \mathrm{~K}$ & $\begin{array}{c}\text { Rate (exp.) } \\
R\left(10^{-3} \mathrm{~s}^{-1}\right)\end{array}$ & $\begin{array}{c}\text { Estimated barrier } \\
E_{A}(\mathrm{eV})\end{array}$ & $\begin{array}{c}\text { Barrier (DFT) } \\
E_{B}(\mathrm{eV})\end{array}$ \\
\hline$R_{\text {step } \rightarrow \text { up }}$ & $1.71 \pm 0.05$ & 1.78 & 2.17 \\
$R_{\text {step } \rightarrow \text { down }}$ & $0.05 \pm 0.02$ & 1.96 & 2.81 \\
$R_{\text {terrace } \rightarrow \text { step }}$ & $4.41 \pm 0.26$ & 1.73 & 1.84 \\
$R_{\text {terrace }}$ & $2.45 \pm 0.68$ & 1.76 & 1.89 \\
\hline \hline
\end{tabular}

abilities. In this way, the microscopic diffusion rate $R_{\text {step } \rightarrow \text { up }}=1.71 \times 10^{-3} \mathrm{~s}^{-1}$ at $568 \mathrm{~K}$ is obtained. It is more than 30 times higher than $R_{\text {step } \rightarrow \text { down }}=0.05 \times 10^{-3} \mathrm{~s}^{-1}$, as obtained by directly analyzing the same STM images. In other words, more than $95 \%$ of all events were recognized as step-up diffusion. Analogously, the rate for hopping from the upper terrace edge site back to the step site (back diffusion) is obtained as $R_{\text {terrace } \rightarrow \text { step }}=4.41 \times 10^{-3} \mathrm{~s}^{-1}$. Independent of the details of the analysis, it is found to be larger than hopping away from the step by a constant factor of 3 . All the measured rates are summarized in Table I. For easier comparison with calculated energy barriers (see below), the experimental rates have been converted to "barrier heights" under the assumption of a pre-exponential factor $\nu=10^{13} \mathrm{~s}^{-1}$ (middle column in Table I). At this point, it should be noted that it is difficult to distinguish between sites with the $\mathrm{H}$ atom on the first dimer and sites with the $\mathrm{H}$ atom on the second dimer of the upper terrace. We therefore combined these two sites in the evaluation of the STM images and refer to them jointly as terrace edge sites whose population is denoted by $P_{\text {te }}$. Depending on the actual potential-energy surface, this might cause, e.g., slight under- and overestimation of the barriers for step-up and intrarow diffusion, respectively. However, when considering the two sites separately in a more complex scenario, we did not find any noteworthy changes.

The hopping rate $R_{\text {step } \rightarrow \text { up }}=1.71 \times 10^{-3} \mathrm{~s}^{-1}$ is nearly one order of magnitude larger than the step depletion rate as determined by means of second-harmonic generation (SHG) measurements. ${ }^{15}$ However, for a comparison one has to keep in mind that, due to back diffusion, this depletion rate must be lower than the microscopic hopping rate $R_{\text {step } \rightarrow \text { up }}$, as determined by means of STM. In order to check the consistency between both experimental methods, we repeated our measurements at a lower temperature, aiming at the determination of the overall step depletion rate. A series of images was acquired at $550 \mathrm{~K}$, and the step depletion as a function of time was recorded. Starting with a step coverage of $95 \%$ at $t=0$, fitting an exponential decay to the step coverage yields an overall step depletion rate of $4 \times 10^{-4} \mathrm{~s}^{-1}$. This result is in good agreement with the value of $1 \times 10^{-4} \mathrm{~s}^{-1}$ extrapolated from the Arrhenius behavior previously found. ${ }^{15}$ We note that the present experimental setup is unsuitable to test the Arrhenius law, since working at higher temperatures would destroy the special initial state (step saturation) that is crucial for our approach.

\section{ANALYSIS OF THE DIFFUSION MECHANISM}

The experimental results on the diffusion rates from the step sites to the lower and upper terraces are surprising when compared to the respective binding energies. The $\mathrm{H}-\mathrm{Si}$ bond is strongest at the step sites. ${ }^{15}$ On the terraces, the hydrogen is found to be weaker bound on the sites above the step edge when compared to the sites below the step edge. ${ }^{25}$ As a consequence, one might expect a higher diffusion barrier and therefore lower diffusion rate for diffusion from the steps to the upper terrace when compared to diffusion from the steps to the lower terrace, in contrast to our experimental observations.

In order to gain an atomistic understanding of this unexpected behavior and its underlying mechanisms, we performed first-principles total-energy calculations for various atomic configurations. As energy reference for our calculations, we chose the situation where the rebonded $\mathrm{Si}$ atoms at the step are completely hydrogen saturated, while all surface $\mathrm{Si}$ atoms on the terraces are free of hydrogen. As known from previous work, step sites bind hydrogen more strongly than any terrace site. ${ }^{15,25}$ Hence, this initial configuration is the thermodynamic ground state at low coverage and low temperature. To investigate the binding energies of an $\mathrm{H}$ atom at other surface sites, we move one $\mathrm{H}$ atom from the rebonded Si step atom to a different surface $\mathrm{Si}$ atom of our choice, keeping the $\mathrm{H}$ coverage constant.

In all calculations, attaching a $\mathrm{H}$ atom to a buckled $\mathrm{Si}$ dimer reduces the buckling angle to a few degrees. We find a slight energetic preference of the $\mathrm{H}$ atom binding to the lower Si atom of the dimer. Moreover, the binding energy of the $\mathrm{H}$ atom is found to decrease monotonously from the lower end to the higher end of the terrace. These findings are in agreement with earlier calculations for pairs of $\mathrm{H}$ atoms, both adsorbed at the same Si dimer on a vicinal surface. ${ }^{25}$

The transition state searches identify a concerted process for $\mathrm{H}$ diffusion across the step, involving rebonding and relaxation of the surface Si atoms (cf. Fig. 3). For diffusion of $\mathrm{H}$ from a step site to the upper terrace, the first stage is the insertion of the $\mathrm{H}$ atom into the $\mathrm{Si}-\mathrm{Si}$ bond of the rebonded $\mathrm{Si}$ atom at the step edge, associated with a barrier at $T_{u 1}$ of $1.36 \mathrm{eV}$. Two further, larger energy barriers, 1.78 and $1.96 \mathrm{eV}$ (relative to the ground state), occur at $T_{u 2}$ and $T_{u 3}$, respectively, when the $\mathrm{H}$ atom climbs up to the first $\mathrm{Si}$ dimer on the upper terrace. Between $T_{u 2}$ and $T_{u 3}$, establishing a weak bond to the $\mathrm{Si}$ atom at the upper ledge introduces an intermediate state. Therefore, the barrier is lower than expected for a hypothetical one-step process. We note that a similar lowering of diffusion barriers by an intermediate has been found in calculations addressing $\mathrm{H}$ diffusion away from a $\mathrm{Si}$-ad-dimer on $\mathrm{Si}(001){ }^{26}$

The potential energy for the diffusion pathway away from the step site $D_{B}$ is displayed in Fig. 4. The weak local minimum associated with the intermediate state for step-up diffusion is illustrated by the ball-and-stick model at the upper right of Fig. 4. The calculations show that the binding energy 


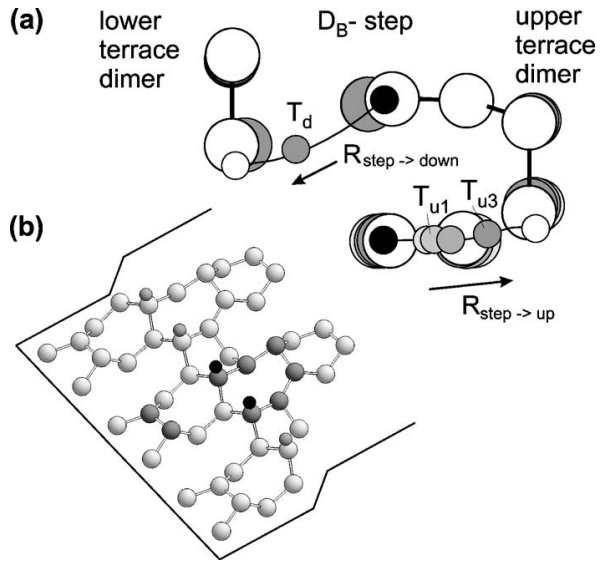

FIG. 3. (a) Calculated atomic configurations during $\mathrm{H}$ diffusion, seen in top view. Small balls are $\mathrm{H}$ atoms and large balls are $\mathrm{Si}$ atoms; multiple images of the atoms, appearing in different shading, indicate concerted motions of the atoms along the diffusion pathway. The lower right part of the figure illustrates step-up diffusion and the upper left part illustrates step-down diffusion. The atoms shown in (a) are highlighted in the perspective view of the step shown in (b).

of the $\mathrm{H}$ atom at the first $\mathrm{Si}$ dimer of the upper terrace (minimum $D_{1}$ in Fig. 4) is significantly reduced by $\approx 0.6 \mathrm{eV}$ compared to the step site, whereas the reduction is only $\approx 0.3 \mathrm{eV}$ for the adjacent $\mathrm{Si}$ dimer on the lower terrace (minimum $D_{-1}$ in Fig. 4). This can be understood from the fact that the $\mathrm{H}$ atom bound to the upper terrace edge breaks a chain of $\pi$ bonds between the dangling bond orbitals of the $\mathrm{Si}$ atoms along the upper edge. Only after overcoming the energy barrier of $2.17 \mathrm{eV}$ at $T_{t}$, the $\mathrm{H}$ atom reaches a more stable po-

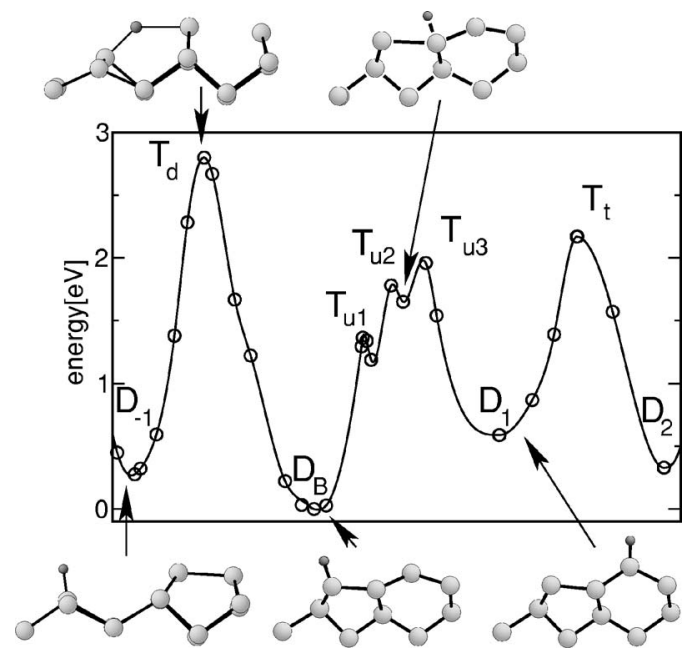

FIG. 4. Calculated potential energy surface for diffusion of one $\mathrm{H}$ atom from a step site (lowest minimum $D_{B}$ ) to the Si dimers on the upper terrace (minima $D_{1}$ and $D_{2}$ in the right part of the plot) or to the Si dimer on the lower terrace (minimum $D_{-1}$ to the left of the plot). The insets below show the geometry of the local minima and the insets above show the transition state for step-down diffusion $T_{d}$ and the metastable intermediate state for step-up diffusion. (All pictures are in side view, small balls are $\mathrm{H}$ atoms, and large balls are $\mathrm{Si}$ atoms.) sition at the second $\mathrm{Si}$ dimer on the upper terrace (minimum $D_{2}$ in Fig. 4). Therefore, we use the value of $2.17 \mathrm{eV}$ when comparing to the experimental apparent activation energy for step-up diffusion in Table I. Similarly, the rate-limiting step for diffusion from the upper terrace to the step is the overcoming of the energy barrier $T_{t}$ coming from the right in Fig. 4 , which amounts to $1.84 \mathrm{eV}$.

Since the two $\mathrm{Si}$ atoms of the $\mathrm{Si}$ dimer on the upper terrace edge are geometrically not equivalent, there are, strictly speaking, two different diffusion pathways for hydrogen atoms from the step to the upper terrace, one leading to the lower $\mathrm{Si}$ atom of the Si terrace edge dimer and one leading to its upper Si atom. By calculating the diffusion pathway and transition states for both cases, we find that the potentialenergy surface is very similar, and energy barriers agree within $0.1 \mathrm{eV}$. In particular, the intermediate state responsible for the lowering of barriers is found to be present in both pathways.

Diffusion from the step to the lower terrace (step-down diffusion) is calculated to be associated with an energy barrier of $2.81 \mathrm{eV}$ at transition state $T_{d}$. Again, diffusion occurs as a concerted process that also involves considerable motion of the $\mathrm{Si}$ atoms. In order to overcome the large distance between initial and final states, the rebonded $\mathrm{Si}$ atom at the step partially follows the motion of the $\mathrm{H}$ atom away from the step. Therefore, its $\mathrm{Si}-\mathrm{Si}$ bond to the ledge is broken at the transition state $T_{d}$ (inset at the upper left of Fig. 4), and only restored after the $\mathrm{H}$ atom has moved to the $\mathrm{Si}$ dimer on the lower terrace (lower left of Fig. 4). In contrast to step-up diffusion, no intermediate stabilization by weak bonds to $\mathrm{Si}$ atoms is possible along this pathway. Thus, the one-step hopping process for step-down diffusion results in a much higher barrier compared to step-up diffusion.

To put the barrier heights into perspective, we also calculate the barrier for $\mathrm{H}$ diffusion along the $\mathrm{Si}$ dimer rows on the terrace. The obtained value of $1.89 \mathrm{eV}$ is larger than the estimate of $1.76 \mathrm{eV}$ derived from the experiments. This is in line with the general trend observed in this study. We therefore conclude that the GGA functional ${ }^{19}$ generally overestimates hydrogen diffusion barriers, while it correctly predicts the relation between barrier heights of different processes. They are summarized in Table I and follow the same order as the experimentally derived activation energies.

\section{SUMMARY}

In summary, we find that $\mathrm{H}$ atoms bound to step sites on vicinal $\mathrm{Si}(001)$ have a strong preference to diffuse to the upper terrace, which is unexpected when judging on the basis of the binding energies with the binding energy for $\mathrm{H}$ at the upper terrace sites being lower than on the lower terrace sites. Density-functional theory calculations show that this is due to a reduction of the energy barrier for diffusion to the upper ledge by stabilization of an intermediate state. We believe that such stabilization is of relevance also for other atomic species diffusing across steps on $\mathrm{Si}(001)$. More importantly, this study shows that simple rules relating diffusion barriers to initial or final state energies are not generally applicable to semiconductor surfaces. 
${ }^{1}$ G. H. Gilmer and P. Bennema, J. Appl. Phys. 43, 1347 (1972).

${ }^{2}$ K. A. Fichthorn and M. Scheffler, Phys. Rev. Lett. 84, 5371 (2000).

${ }^{3}$ B. Voigtländer, Th. Weber, P. Šmilauer, and D. E. Wolf, Phys. Rev. Lett. 78, 2164 (1997).

${ }^{4}$ E. Kim, C. W. Oh, and Y. H. Lee, Phys. Rev. Lett. 79, 4621 (1997).

${ }^{5}$ J. H. G. Owen, D. R. Bowler, C. M. Goringe, K. Miki, and G. A. D. Briggs, Phys. Rev. B 54, 14153 (1996).

${ }^{6}$ M. McEllistrem, M. Allgeier, and J. J. Boland, Science 279, 545 (1998).

${ }^{7}$ E. Hill, B. Freelon, and E. Ganz, Phys. Rev. B 60, 15896 (1999).

${ }^{8}$ A. Vittadini, A. Selloni, and M. Casarin, Surf. Sci. 289, L625 (1993).

${ }^{9}$ C. J. Wu, I. V. Ionova, and E. A. Carter, Phys. Rev. B 49, 13488 (1994).

${ }^{10}$ P. Nachtigall and K. D. Jordan, J. Chem. Phys. 102, 8249 (1995).

${ }^{11}$ P. Kratzer, E. Pehlke, M. Scheffler, M. B. Raschke, and U. Höfer, Phys. Rev. Lett. 81, 5596 (1998).

${ }^{12}$ M. B. Raschke and U. Höfer, Appl. Phys. B: Lasers Opt. 68, 649 (1999).

${ }^{13}$ M. Dürr, M. B. Raschke, E. Pehlke, and U. Höfer, Phys. Rev.
Lett. 86, 123 (2001).

${ }^{14}$ M. Dürr and U. Höfer, Surf. Sci. Rep. 61, 465 (2006).

${ }^{15}$ M. B. Raschke and U. Höfer, Phys. Rev. B 59, 2783 (1999).

${ }^{16}$ M. Dürr, Z. Hu, A. Biedermann, U. Höfer, and T. F. Heinz, Phys. Rev. B 63, 121315(R) (2001).

${ }^{17}$ D. J. Chadi, Phys. Rev. Lett. 59, 1691 (1987).

${ }^{18}$ M. Bockstedte, A. Kley, J. Neugebauer, and M. Scheffler, Comput. Phys. Commun. 107, 187 (1997).

${ }^{19}$ J. P. Perdew, J. A. Chevary, S. H. Vosko, K. A. Jackson, M. R. Pederson, D. J. Singh, and C. Fiolhais, Phys. Rev. B 46, 6671 (1992).

${ }^{20}$ M. Fuchs and M. Scheffler, Comput. Phys. Commun. 116, 67 (1999).

${ }^{21}$ I. V. Ionova and E. A. Carter, J. Chem. Phys. 98, 6377 (1993).

${ }^{22}$ J. Tersoff and D. R. Hamann, Phys. Rev. B 31, 805 (1985).

${ }^{23}$ M. Dürr, Z. Hu, A. Biedermann, U. Höfer, and T. F. Heinz, Phys. Rev. Lett. 88, 046104 (2002).

${ }^{24}$ M. Dürr, A. Biedermann, Z. Hu, U. Höfer, and T. F. Heinz, Science 296, 1838 (2002).

${ }^{25}$ E. Pehlke and P. Kratzer, Phys. Rev. B 59, 2790 (1999).

${ }^{26}$ D. R. Bowler, Phys. Rev. B 67, 115341 (2003). 